\title{
Engulfment and cell motility protein 1 potentiates diabetic cardiomyopathy via Rac-dependent and Rac-independent ROS production
}

\author{
Masao Kakoki, ${ }^{1}$ Edward M. Bahnson, ${ }^{1,2}$ John R. Hagaman, ${ }^{1}$ Robin M. Siletzky, ${ }^{2}$ Ruriko Grant, ${ }^{1}$ \\ Yukako Kayashima, ${ }^{1}$ Feng Li, ${ }^{1}$ Esther Y. Lee, ${ }^{1}$ Michelle T. Sun, ${ }^{1}$ Joan M. Taylor, Jessica C. Rice, ${ }^{3}$ \\ Michael F. Almeida, ${ }^{3}$ Ben A. Bahr, ${ }^{3}$ J. Charles Jennette, ${ }^{1}$ Oliver Smithies, ${ }^{1}$ \\ and Nobuyo Maeda-Smithies ${ }^{1}$ \\ 'Department of Pathology and Laboratory Medicine, University of North Carolina at Chapel Hill, Chapel Hill, North Carolina, \\ USA. 'Department of Surgery, Division of Vascular Surgery, and Department of Cell Biology and Physiology, University of \\ North Carolina at Chapel Hill, Chapel Hill, North Carolina, USA. ${ }^{3}$ Biotechnology Research and Training Center, University of \\ North Carolina at Pembroke, Pembroke, North Carolina, USA.
}

\begin{abstract}
Engulfment and cell motility protein 1 (ELM01) is part of a guanine nucleotide exchange factor for Ras-related C3 botulinum toxin substrate (Rac), and ELM01 polymorphisms were identified to be associated with diabetic nephropathy in genome-wide association studies. We generated a set of Akita Ins $2^{\text {c96r }}$ diabetic mice having 5 graded cardiac mRNA levels of ELMO1 from $30 \%$ to $200 \%$ of normal and found that severe dilated cardiomyopathy develops in ELMO1-hypermorphic mice independent of renal function at age 16 weeks, whereas ELM01-hypomorphic mice were completely protected. As ELMO1 expression increased, reactive oxygen species indicators, dissociation of the intercalated disc, mitochondrial fragmentation/dysfunction, cleaved caspase-3 levels, and actin polymerization increased in hearts from Akita mice. Cardiomyocyte-specific overexpression in otherwise ELM01-hypomorphic Akita mice was sufficient to promote cardiomyopathy. Cardiac Rac1 activity was positively correlated with the ELMO1 levels, and oral administration of a pan-Rac inhibitor, EHT1864, partially mitigated cardiomyopathy of the ELMO1 hypermorphs. Disrupting Nox4, a Rac-independent NADPH oxidase, also partially mitigated it. In contrast, a pan-NADPH oxidase inhibitor, VAS3947, markedly prevented cardiomyopathy. Our data demonstrate that in diabetes mellitus ELMO1 is the "rate-limiting" factor of reactive oxygen species production via both Rac-dependent and Rac-independent NADPH oxidases, which in turn trigger cellular signaling cascades toward cardiomyopathy.
\end{abstract}

Authorship note: OS is deceased.

Conflict of interest: The authors have declared that no conflict of interest exists.

Copyright: (c) 2019, American Society for Clinical Investigation.

Submitted: January 22, 2019

Accepted: May 8, 2019

Published: June 20, 2019

Reference information: /CI Insight. 2019;4(12):e127660. https://doi. org/10.1172/jci.insight.127660

\section{Introduction}

Engulfment and cell motility protein 1 (ELMO1) physically interacts with the dedicator of cytokinesis 180 (DOCK180), a member of the guanine nucleotide exchange factors (GEFs), to promote small GTPase Ras-related $\mathrm{C} 3$ botulinum toxin substrate (Rac), and was first discovered as a gene required for phagocytosis of apoptotic cells and cell motility $(1,2)$. In a genome-wide case control association study, Shimazaki et al. found that a single nucleotide polymorphism in the 18th intron of the ELMO1 gene is strongly associated with diabetic nephropathy in Japanese (3). Since then the association has been observed between the polymorphisms in the ELMO1 gene and diabetic nephropathy in Whites (4, 5), Chinese (6), African Americans (7), Pima Indians (8), South Indians (9), and Iranians (10).

In our previous work, we demonstrated the causal relationships between the genetic variations in the expression of Elmo1 and the severity of nephropathy by generating Akita type 1 diabetic mice having 5 graded genetic levels of Elmo1 expression by replacing the 3' untranslated region (3'-UTR) of Elmo1 with either an unstable Fos $3^{\prime}$-UTR or a stable bovine growth hormone $3^{\prime}$-UTR $(b G H)(11,12)$. Akita diabetic males develop diabetic nephropathy including albuminuria, glomerulosclerosis, and the thickening of glomerular basement membrane, and our experiment showed that the severity of nephropathy increased 
in parallel with the expression of Elmo1, despite blood pressure or plasma levels of glucose or insulin not being significantly altered (12). Direct correlations between Elmo1 expression levels and the severity of nephropathy as well as the level of plasma ROS in these mice led us to hypothesize that genetic variations of Elmo1 expression may also affect severity of diabetic complications in other organs.

Metabolic dysregulation caused by diabetes increases the risk of developing cardiovascular diseases (13, 14). Because atherosclerosis and hypertension are highly common conditions that influence cardiovascular diseases and also interact with diabetes, cardiac complications of diabetes have not been clearly defined. However, more recently, diabetic cardiomyopathy has become recognized as a clinical entity of global cardiac dysfunction that is associated with diabetes mellitus but not caused by hypertensive, coronary, or valvular heart disease (15). Studies have demonstrated that it is frequently present ( $\sim 60 \%$ patients with type 2 diabetes) and associated with premature sudden death in diabetic patients. Various animal models of diabetes have also confirmed the development of diabetes-induced cardiomyopathy with diastolic dysfunction $(13,14)$.

In the current study, we evaluated the morphological and functional changes in the hearts of Akita mice with 5 graded levels of ELMO1. The high expression of Elmo1 in Akita diabetic mice caused severely impaired cardiac contractile function and significant dilation of the internal diameter of the left ventricle. In contrast, Akita diabetic mice with lower-than-normal expression of Elmo1 did not show the contractile dysfunction. Examination of plausible pathogenic mechanisms, whereby high genetic levels of ELMO1 exacerbate dilated cardiomyopathy, revealed that both Rac-dependent NADPH oxidases and Rac-independent NADPH oxidase 4 (NOX4) play an important role in the development of diabetic cardiomyopathy. These findings suggest that suppressing ELMO1 or inhibiting both Rac-dependent and Rac-independent NADPH oxidases could be promising options for preventing diabetic cardiomyopathy.

\section{Results}

Akita diabetic mice having 5 genetically different levels of ELMO1. The wild-type (WT), hypomorphic (L), and hypermorphic $(\mathrm{H})$ alleles for the Elmo1 gene are illustrated in Figure 1A. The 3'-UTR of the Elmo ${ }^{L}$ allele was replaced with that of the Fos gene, resulting in a reduced mRNA half-life. The Elmo1 $1^{L}$ allele was designed to be switched after Cre-loxP recombination to an Elmo ${ }^{H}$ allele in which the use of $3^{\prime}$-UTR of $b G H$ increases the half-life of Elmo1 mRNA (12). To determine the roles of ELMO1 in diabetic complications in hearts, diabetic mice globally having 5 graded expression levels of the Elmo1 gene were obtained by the 5 combinations of the Elmo1 ${ }^{+}, E l m o 1^{L}$, and $E l m o 1^{H}$ alleles on a background of heterozygous diabetogenic Akita mutation in the insulin 2 gene (Ins2). At age 16 weeks, the hearts of the resulting Akita diabetic male

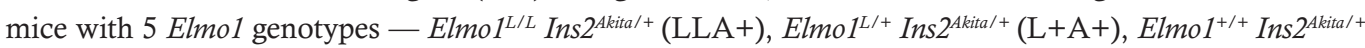
(WTA+), Elmo1 ${ }^{H /+}$ Ins2 $2^{\text {Akital+ }}(\mathrm{H}+\mathrm{A}+)$, and Elmo1 ${ }^{H / H} I_{n s} 2^{A k i t a /+}(\mathrm{HHA}+)$ - had Elmo1 mRNA expression graded in 5 steps from approximately $30 \%$ to $200 \%$ (Figure 1B) and ELMO1 protein levels graded in 5 steps from $30 \%$ to approximately 190\% compared with those of the WTA+ mice (Figure 1C). Cardiac ELMO1 protein levels in diabetic WTA+ mice were twice the levels in nondiabetic WT mice (Supplemental Figure 1A; supplemental material available online with this article; https://doi.org/10.1172/jci. insight.127660DS1). In contrast, the protein levels of ELMO2 and ELMO3 were decreased as the expression of Elmo1 increased (Figure 1, D and E), while levels of other components of the ELMO-DOCK complex, including DOCK180 and DOCK2, did not significantly differ (Supplemental Figure 1, B and C).

In addition, the heart weights normalized by tibia length or body weight were not different (Figure $1 \mathrm{~F}$ and Supplemental Figure 1D). In contrast, the heart rates in the HHA+ mice were significantly less than in the WTA+ mice (Figure 1G). ELMO1 is the part of a Rac-GEF converting Rac-GDP into Rac-GTP. We, therefore, determined the Rac1 activity in the hearts of the Akita diabetic mice as the protein amount of Rac1 that can bind to the 21 binding domain of the p21-activated kinase 1. Rac1-GTP in Western blot (Figure $1 \mathrm{H}$ ) showed that the levels of Rac1 activity directly paralleled with the genetically graded levels of Elmo1 expression. Total amount of Rac1 protein was not altered (Supplemental Figure 1E); thus, the amount of ELMO1 was the direct determinant of the Rac activity in the hearts of Akita diabetic mice.

Plasma levels of glucose and arterial pressure at 16 weeks of age were not significantly different as we previously reported (ref. 12 and Supplemental Figure 2, A and B). Similarly, the plasma hemoglobin A1C levels, a long-term index of glycemic control, were not different among the 5 Elmo1 genotypes (Supplemental Figure 2C). Thyroid hormones triiodothyronine and thyroxine may affect cardiac function, but their plasma levels were not significantly different among Akita mice having the 5 Elmo1 genotypes, excluding the possibility of their involvement (Supplemental Figure 3, A and B). 
A WT

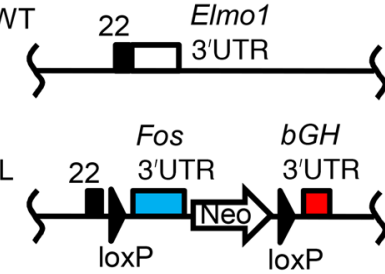

$\mathrm{H}$

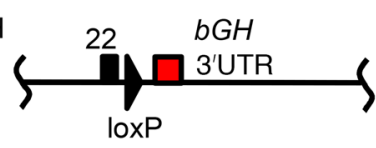

C

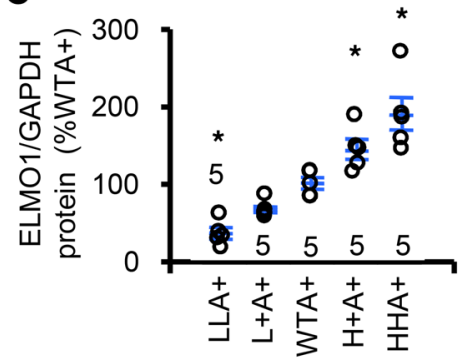

E

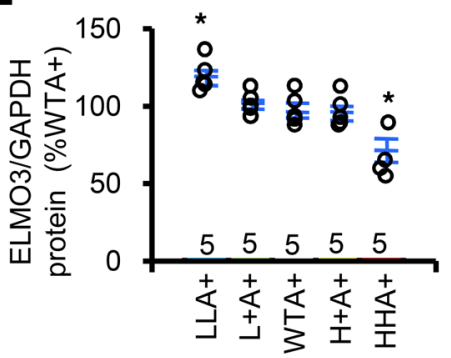

G

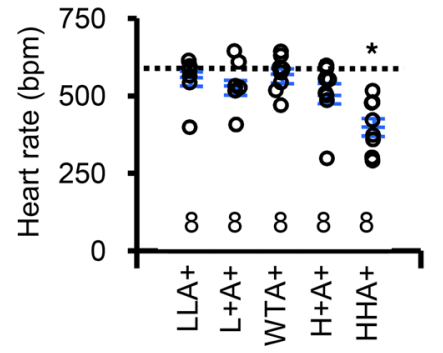

B

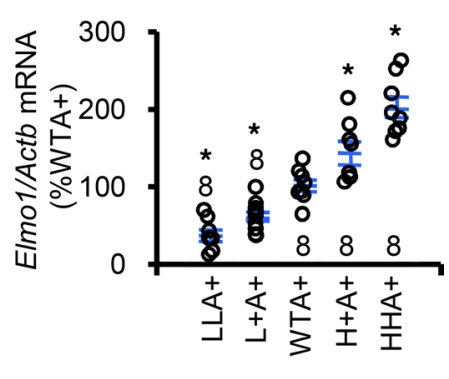

D

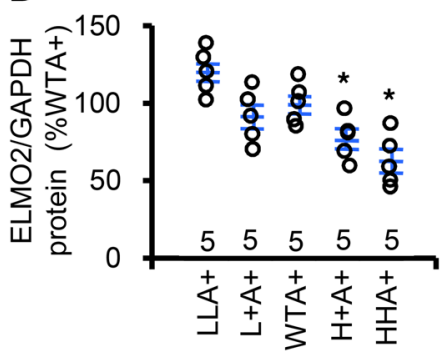

$\mathbf{F}$

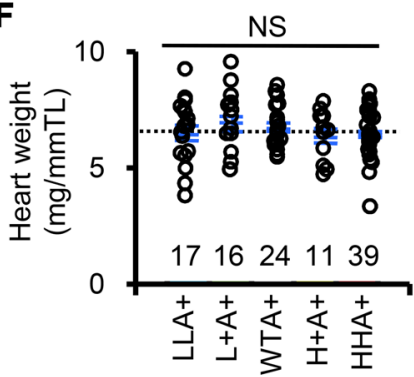

H

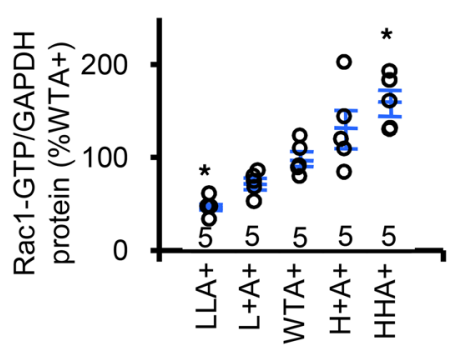

Figure 1. Basic cardiac phenotypes of the Akita diabetic mice having 5 graded expression levels of Elmo1 at age 16 weeks. LLA+, Elmo1/L Ins2 $2^{A k i t a /+} ; \mathrm{L}+\mathrm{A}+, E^{2} \operatorname{mo1}^{L /+} \operatorname{Ins} 2^{\text {Akita/+ }}$; WTA+, Elmo1+/+ Ins2 $2^{A k i t a /+} ; \mathrm{H}+\mathrm{A}+$, $\mathrm{Elmo}^{1 /++}$ Ins2 $2^{\mathrm{Akita} /+} ; \mathrm{HHA}+, \mathrm{Elmo}^{\mathrm{H} / \mathrm{H}}$

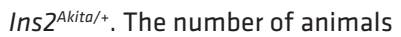
studied is shown in each figure. Data are expressed as mean \pm SEM. Comparisons were done with ANOVA including the additional data set. ${ }^{*} P<0.05$ vs. WTA+ mice by Tukey-Kramer Honestly Significant Differences test. NS, not significantly different among the 5 groups. (A) Top, the WT allele for Elmo1. Coding portion of the exon 22 and the endogenous 3 '-UTR of Elmo1 are shown as a black box and white box, respectively. Middle, the low-expressing $(\mathrm{L})$ allele. The stability of the Elmo1 mRNA is now controlled by the destabilizing 3'-UTR of Fos (blue box). Bottom, the high-expressing $(\mathrm{H})$ allele. The $L$ allele can be converted into the $\mathrm{H}$ allele by Cre-loxP recombination. The stability of the Elmo1 mRNA is now controlled by the stabilizing 3'-UTR of bGH (red box). (B) Cardiac Elmo1 mRNA levels normalized by mRNA of $\beta$-actin (Actb). (C-E) Protein levels of ELMO1, ELMO2, and ELMO3 in each animal were normalized with the GAPDH level. (F) Heart weight normalized by tibia length (TL) and (G) heart rate. Dotted lines indicate nondiabetic WT levels. (H) Activity of Rac1 quantitated by Rac1-GTP signals.

High ELMO1 accelerates and low ELMO1 prevents dilated cardiomyopathy in Akita diabetic mice. Cross sections of hearts showed that both chamber dilation and collagen fiber accumulation (blue color with AZAN trichrome staining) dramatically intensified as the expression of Elmo1 augmented (Figure 2A). Echocardiograms revealed that the ejection fraction of the left ventricle was significantly reduced in the $\mathrm{HHA}+$ and $\mathrm{H}+\mathrm{A}+$ mice as compared with the WTA+ mice at the age of 16 weeks (Figure 2, B and C). In the reverse direction, the left ventricular ejection fraction in the LLA+ mice was significantly greater than in the WTA+ mice and equivalent to that in nondiabetic WT mice (dotted lines in Figure 2C). Likewise, the left ventricular posterior wall in diastole of the HHA+ heart was significantly thinner than in the $\mathrm{WTA}+$ mice (Figure 2D). The internal diameter of the left ventricle in diastole was significantly greater in the $\mathrm{HHA}+$ and $\mathrm{H}+\mathrm{A}+$ mice than in the WTA+ mice (Figure 2E). The E-wave deceleration rate (EWDR) of the mitral flow was significantly less in the HHA+ mice than in the WTA+ mice (Figure 2F). The isovolumic relaxation time (IVRT) of the left ventricle was significantly greater in the HHA+ mice than in the WTA+ mice (Figure 2G). The early tissue Doppler velocity (E') was significantly less in the HHA+ and $\mathrm{H}+\mathrm{A}+$ mice than in the WTA+ mice (Figure $2 \mathrm{H}$ ). There was no indication of valvular or coronary diseases throughout the echocardiographic study. 


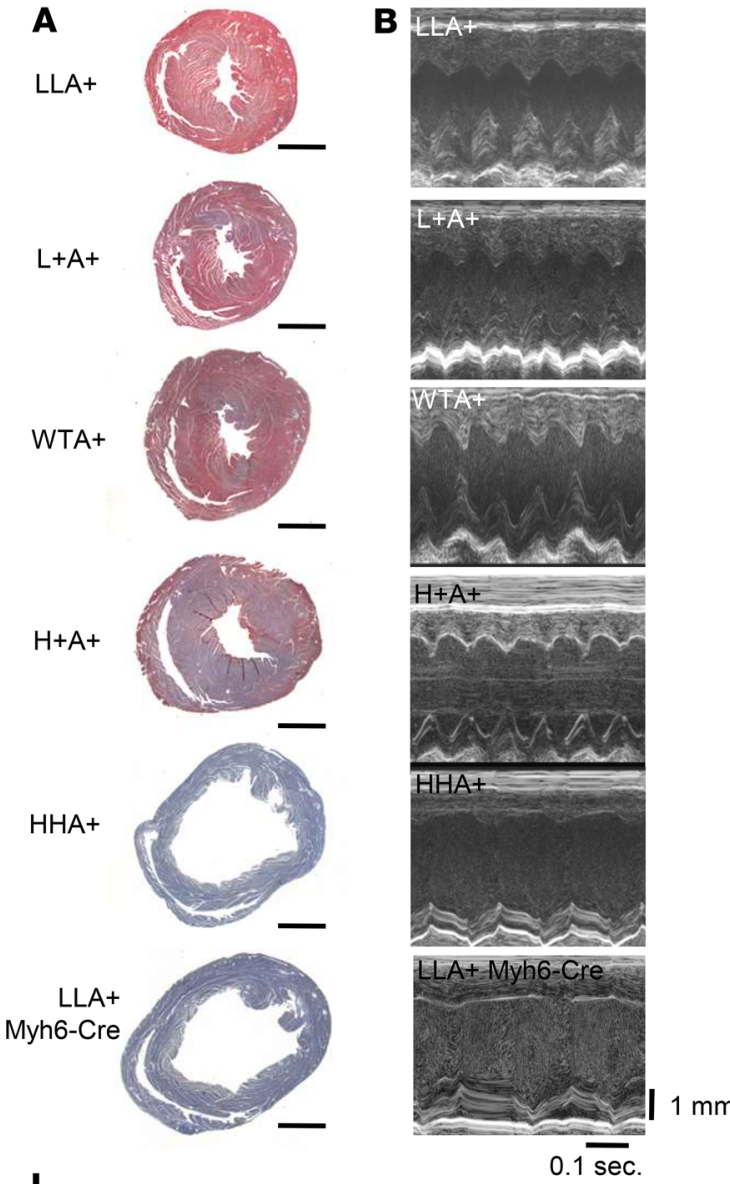

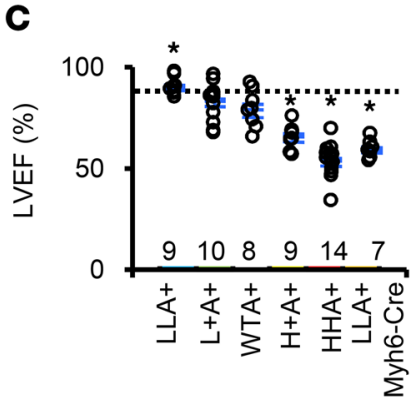
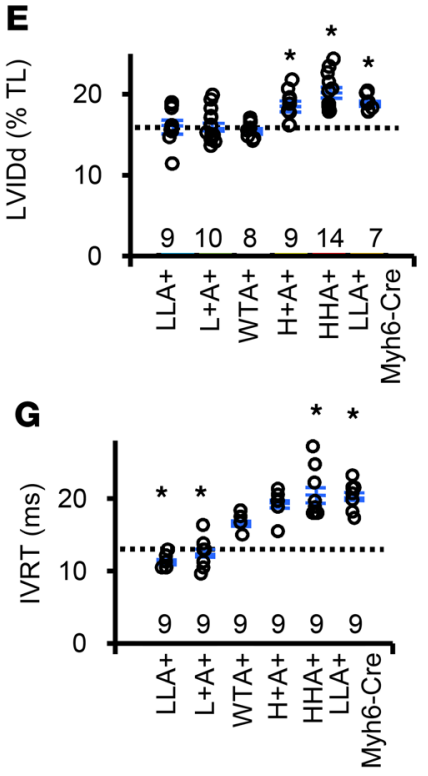

D

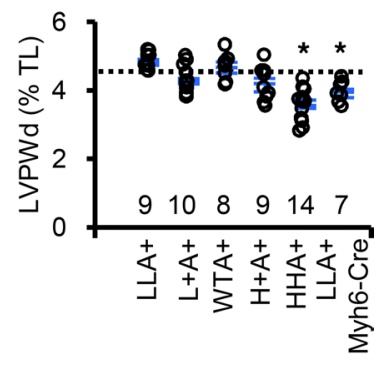

$\boldsymbol{F}$
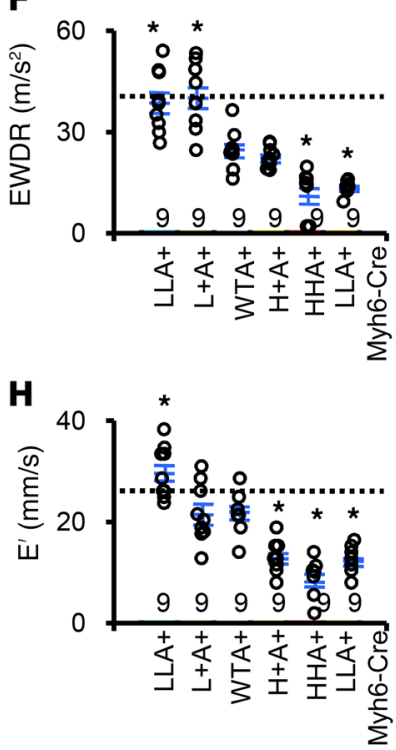

$\mathrm{HHA}+$

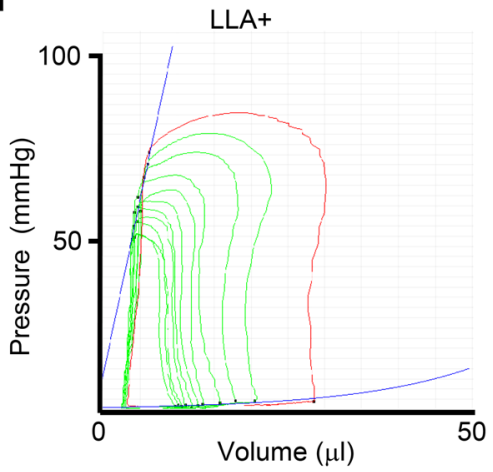

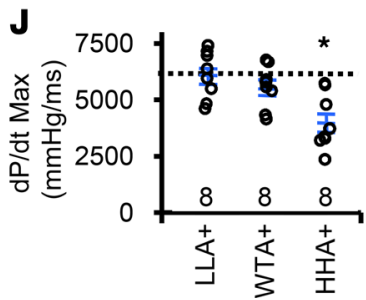

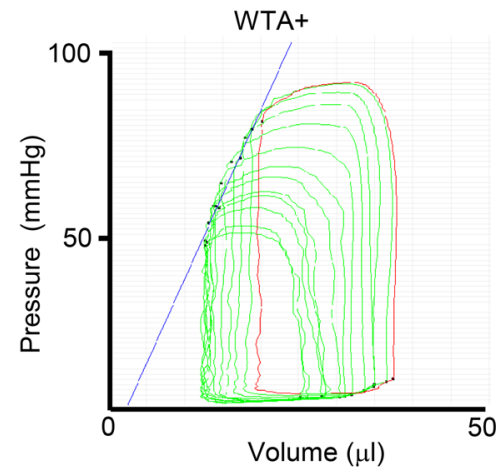

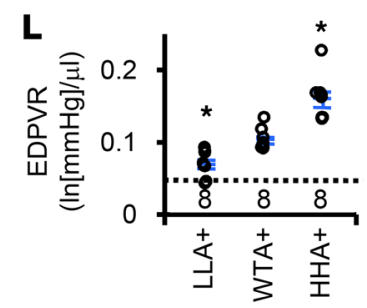
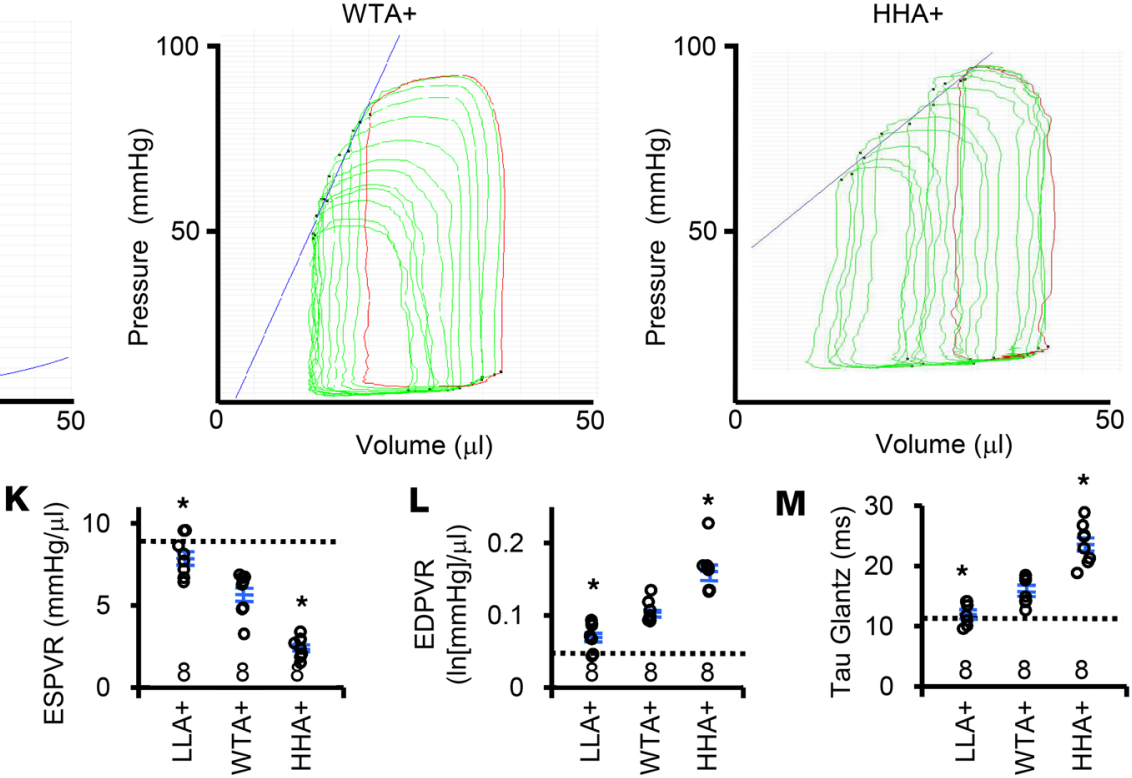

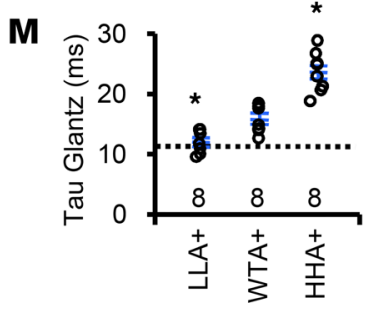

Figure 2. Histology and cardiac functions with echocardiography and pressure-volume loop study. "LLA+Myh6-Cre" indicates LLA+ mice with the cardiomyocyte-specific Cre transgene; dotted lines indicate nondiabetic WT levels. The number of animals studied is shown in each figure. Data are expressed as mean \pm SEM. Comparisons were done with 1-way ANOVA including the additional data set. ${ }^{*} P<0.05$ vs. WTA+ mice by Tukey-Kramer Honestly Significant Differences test. (A) AZAN trichrome staining of the heart. Scale bar: $1 \mathrm{~mm}$. (B) Representative M-mode echocardiograms. (C) Ejection fraction of the left ventricles (LVEF). (D) Thickness of the left ventricular posterior wall in diastole (LVPWd). Values are expressed as percentage of TL. (E) Internal diameter of the left ventricle in diastole (LVIDd) as percentage of TL. (F) E-wave deceleration rate (EWDR) of the mitral flow. (G) The isovolumic relaxation time (IVRT) of the left ventricle. (H) Early tissue Doppler velocity (E'). (I) Representative PV loops. (J) $d P / d t$ max. (K) End-systolic pressure-volume relationship (ESPVR). (L) End-diastolic pressure-volume relationship (EDPVR). (M) Tau Glantz. 
There was no significant difference in the heart weight normalized by body weight, systolic blood pressure, heart rate, and plasma glucose among nondiabetic mice (Supplemental Figure 4, A-D). No remarkable alteration in the heart was detectable in histology (Supplemental Figure 4E). The detrimental effects on the cardiac function were not observed in echocardiography in nondiabetic mice with high Elmo1 expression (Supplemental Figure 4, F-K). Overall, 2-way ANOVA showed strong interactions between Elmo1 genotype and diabetes in the ejection fraction, wall thickness, and internal diameter (Supplemental Table 1), suggesting that ELMO1 and diabetes enhance the cardiac dysfunction synergistically.

To further examine the mechanical properties of the hearts of Akita mice, we next performed a pressure-volume (PV) loop study (Figure 2I). The parameters indicative for the systolic function, including $d P / d t$ max and end-systolic pressure-volume relationship (ESPVR), were significantly smaller in the $\mathrm{HHA}+$ mice than in the WTA+ mice (Figure 2, J and $\mathrm{K}$ ). In the reverse direction, these parameters for the systolic functions in the LLA+ mice were significantly higher than in the WTA+ mice. In contrast, the parameters indicative for the diastolic function end-diastolic pressure-volume relationship (EDPVR) and Tau Glantz value were significantly higher in the HHA+ mice than in the WTA+ mice (Figure 2, L and M). Again, in the reverse direction, the EDPVR and Tau Glantz in the $\mathrm{LLA}+$ mice were lower than in the WTA+ mice. This indicates that ELMO1 diminishes both systolic and diastolic function in Akita diabetic hearts. There was no detectable difference in $d P / d t \max$, ESPVR, EDPVR, or Tau Glantz among nondiabetic mice with different ELMO1 expression (Supplemental Figure 4, L-O).

Cardiomyocyte-specific overexpression of Elmo1 is sufficient to cause dilated cardiomyopathy in the HHA+ mice. We previously showed that the glomerulosclerosis and renal excretory dysfunction were markedly enhanced in the HHA+ mice in comparison with the WTA+ mice at the age of 40 weeks (12). This raises a possibility that the cardiac dysfunction in the $\mathrm{HHA}+$ mice may be due to renal excretory dysfunction and fluid retention. However, at the age of 16 weeks, the renal histology and the plasma cystatin $\mathrm{C}$ levels were not significantly different among Akita mice having 5 Elmo1 genotypes, suggesting that the kidney functions of these mice were not yet affected (Supplemental Figure 5, A-D). Thus, development of cardiac dysfunction appears to start before renal dysfunction.

To confirm that the effects of high ELMO1 leading to cardiac dysfunction are cardiomyocyte intrinsic and not secondary to other peripheral tissue damage or other cell types in the heart, we generated mice overexpressing Elmo1 specifically in the cardiomyocyte by introducing a Cre-loxP recombinase driven by Myh6 promoter into the LLA+ mice. In the resulting Elmo1 ${ }^{L / L}$ Myh6-Cre Ins $2^{A k i t a /+}$ mice, the Elmo1 $1^{L}$ allele switches to an Elmo1 ${ }^{H}$ allele only in cardiomyocytes, but cells in the rest of the body remain ELMO1 hypomorphic. Echocardiograms revealed that cardiomyocyte-specific overexpression of Elmo1 is sufficient to recapitulate the dilated cardiomyopathy phenotype (Figure 2, A-H). This clearly demonstrates that enhanced cardiomyopathy in the HHA+ mice does not require global overexpression of Elmo1 but is the result of cardiomyocyte cell-autonomous ELMO1 effects.

Dissociated intercalated discs, reduced cardiac connexin43, and elongated QTc intervals. Transmission electron microscopy (TEM) revealed dissociation of the intercalated discs in the HHA+ and WTA+ mice that was not present in the LLA+ mice (Figure 3, A and B). The immunoreactivity of connexin43, the principal component of the gap junction in the intercalated disc (16), decreased as the expression of Elmo1 increased (Figure 3C). Intercalated discs connect individual cardiomyocytes and propagate electrochemical communication so that the heart works as a single functional organ. Structural stability of the intercalated disc is regulated by phosphorylation of serine residues of connexin 43 (17), and the phospho-Ser368 is necessary for myocardial conduction during metabolic stress (18).

Immunoblot analysis showed that total connexin43 protein in the whole heart was decreased as the expression of Elmo1 increased in Akita mice accompanied by a more dramatic reduction of Ser368-phosphorylated connexin43 (Figure 3, D and E). The cardiac protein levels of small G protein signaling modulator 3 (SGSM3), which is reported to be upregulated upon ROS exposure and induces connexin43 turnover via lysosomal degradation in cardiomyocytes (19), were increased as the expression of Elmo1 augmented in Akita mice (Figure 3F).

The functional outcome of the dissociation of the intercalated disc in the electrocardiogram was a significant elongation of the QT interval in the HHA+ mice (Figure 3G), even after the correction by RR intervals using Bazzette's formula (QTcB). In comparison, QT intervals of the LLA+ mice were shorter than WTA+ 
A
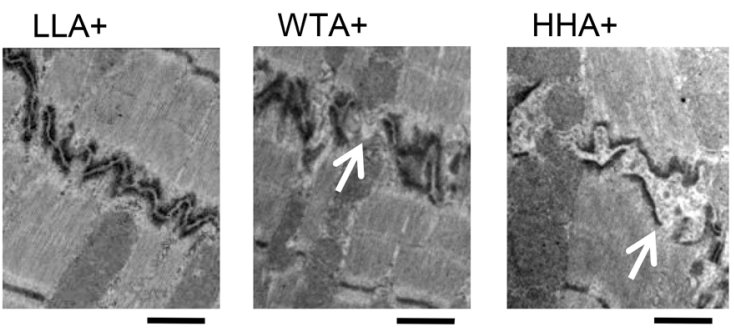

B

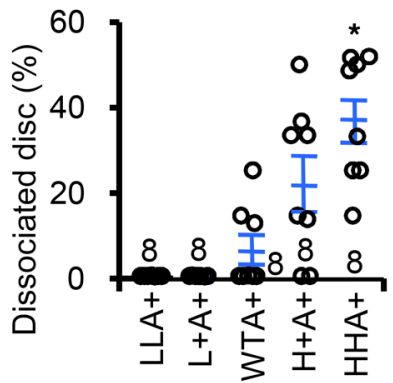

C
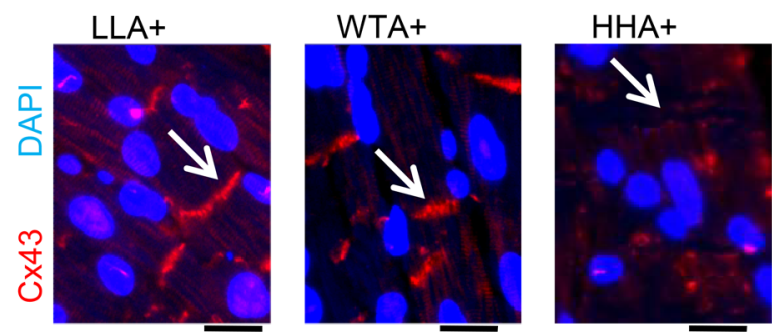

D

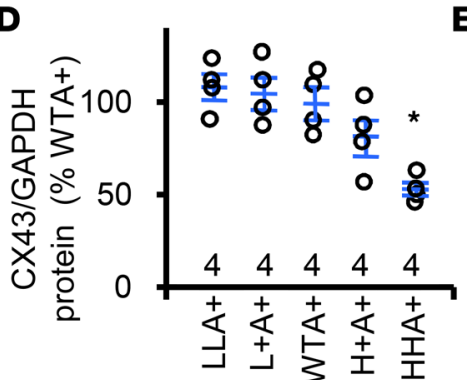

E

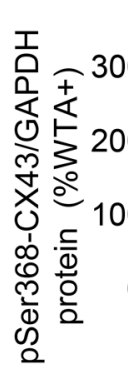

G

$\mathbf{F}$

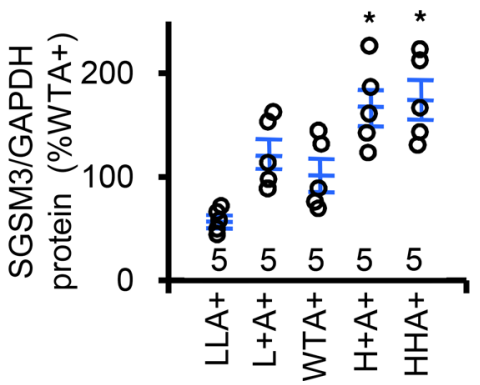

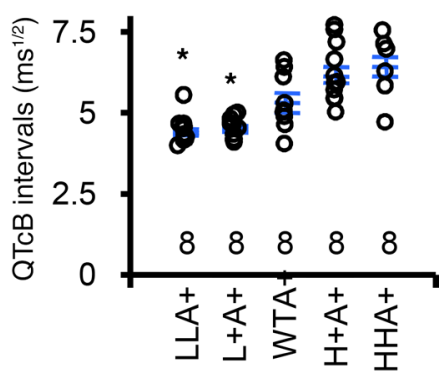

Figure 3. Dissociation of the intercalated disc and impaired electric conduction of heart muscle cells. The number of animals studied is shown in each figure. Data are expressed as mean \pm SEM Comparisons were done with 1-way ANOVA including the additional data set. ${ }^{*} P<0.05$ vs. WTA+ by Tukey-Kramer Honestly Significant Differences test. NS, not significantly different among the 5 groups. (A) Representative images of the intercalated disc in the hearts of LLA+, WTA+, and HHA+ mice as shown by TEM. White arrows indicate the dissociation of the intercalated disc. Scale bar: $1 \mu \mathrm{m}$. (B) Frequency of the dissociation of the intercalated disc. Percentage of discs with dissociated gap junctions per at least 30 discs in each animal. (C) Representative images for the immunofluorescence for connexin43 (Cx43). DAPI shows nuclei. The immunoreactivity for connexin43 was diminished in the intercalated discs in the $\mathrm{HHA}+$ heart. Arrows indicate intercalated discs. Scale bar: $10 \mu \mathrm{m}$. (D-F) Protein levels of (D) total connexin43, (E) Ser368-phosphorylated connexin43, and (F) small G protein signaling modulator 3 (SCSM3) were normalized with GAPDH protein and expressed relative to the mean value in the WTA+ as $100 \%$. (C) QT intervals in the electrocardiogram after correction by RR intervals using Bazzette's formula (QTcB).

mice and indistinguishable from those in the WT nondiabetic C57BL/6 mouse. The PR intervals and the QRS complex were not different among the 5 Elmo1 genotypes (Supplemental Figure 6, A and B).

These data demonstrate that the mechanical and functional dissociations of the intercalated disc observed in the Akita diabetic mice are strongly affected by the amounts of ELMO1, ranging from almost the same in nondiabetic WT mice as in LLA+ mice to severe difference in HHA+ mice. These pathological changes are likely to play causative roles in the development of systolic dysfunction in the ELMO1-hypermorphic Akita mice.

Mitochondrial dysfunction and fragmentation. Cardiac content of ATP and citrate synthase activity, both of which reflect mitochondrial function, were lower in the HHA+ mice than the WTA+ mice (Figure 4, A and B). 
A

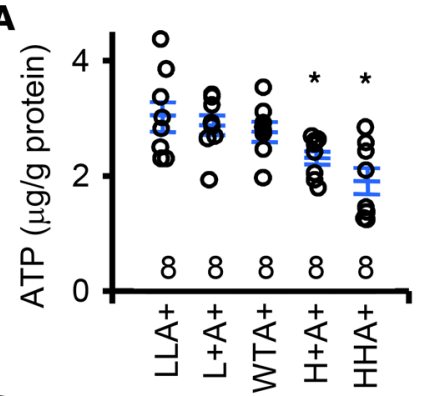

C

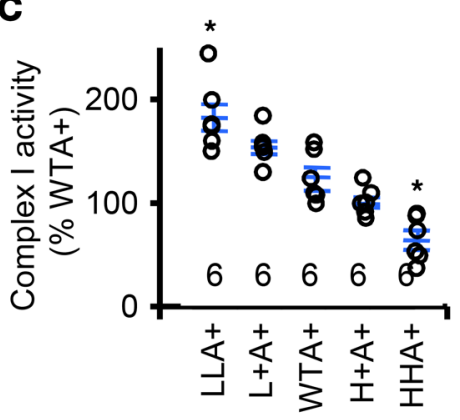

$\mathbf{E}$
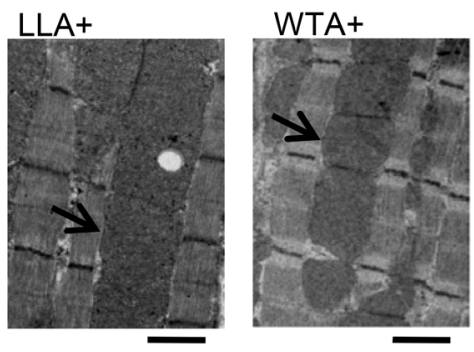

$\mathbf{F}$

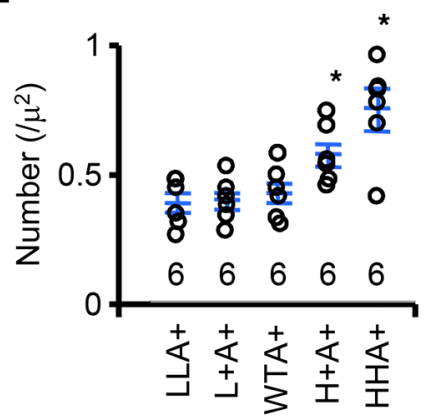

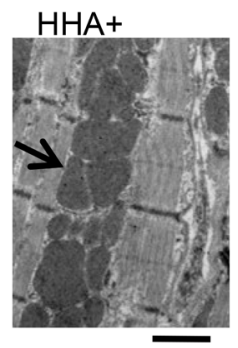

B

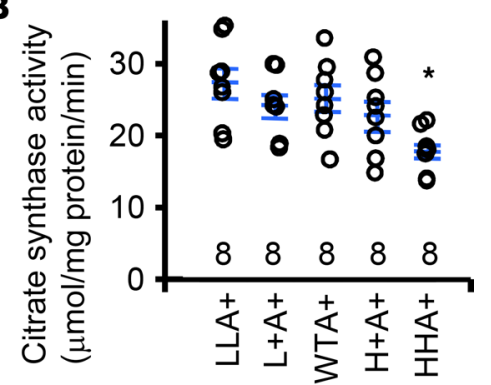

D

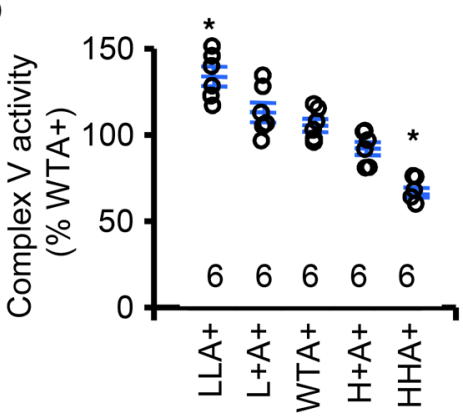

G

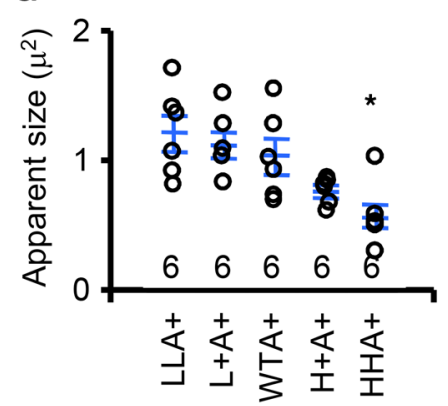

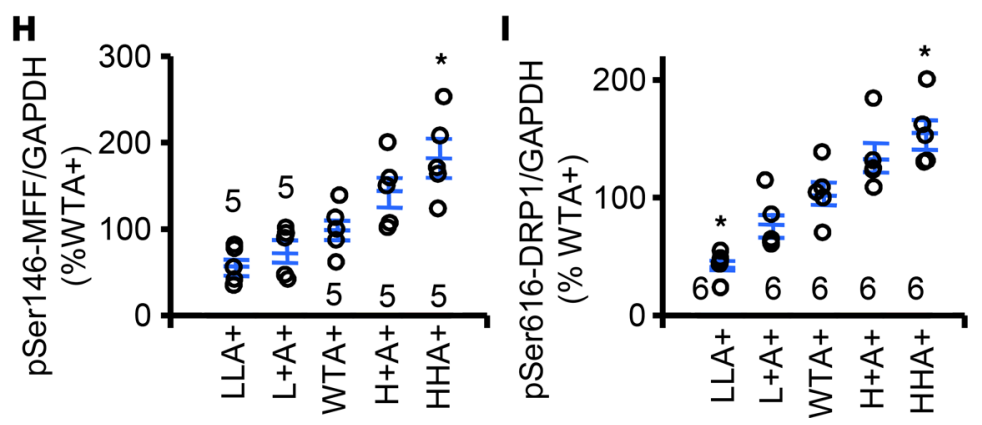

More specifically, the cardiac activities of mitochondrial complexes I, IV, and V were lower in the HHA+ mice than the WTA+ mice (Figure 4, C and D, and Supplemental Figure 7A). However, the cardiac activities of mitochondrial complexes II and III were not different among the 5 groups of Akita mice (Supplemental Figure 7, B and C). TEM also revealed that the fragmentation of the mitochondria was more prominent in the HHA+ 
A

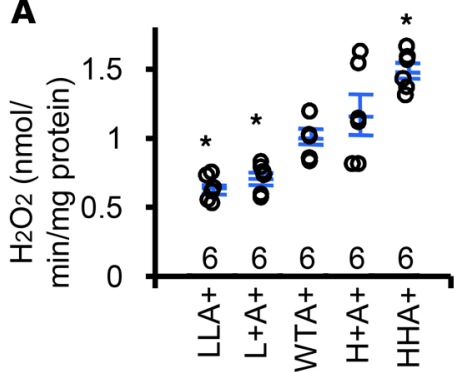

C

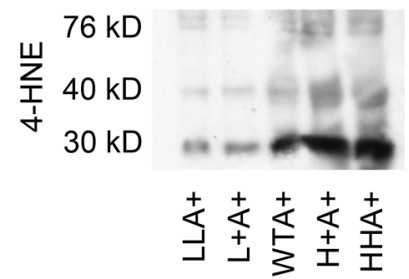

E

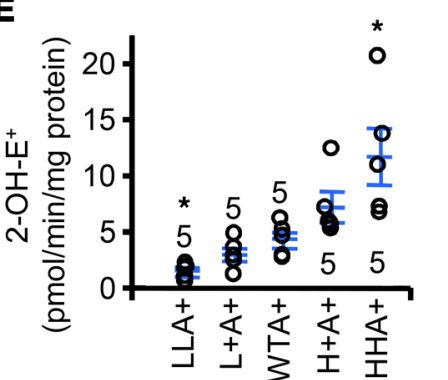

G

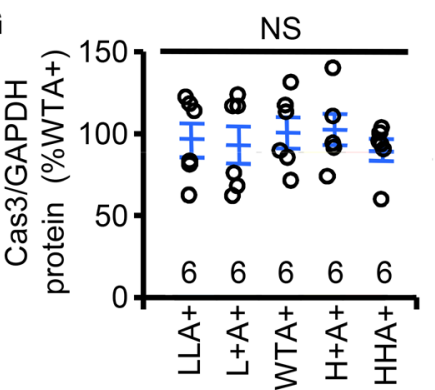

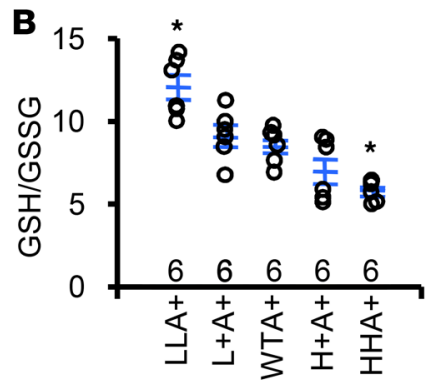

D

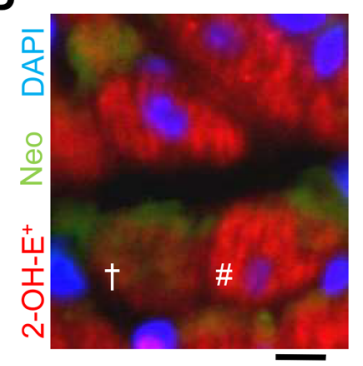

$\mathbf{F}$
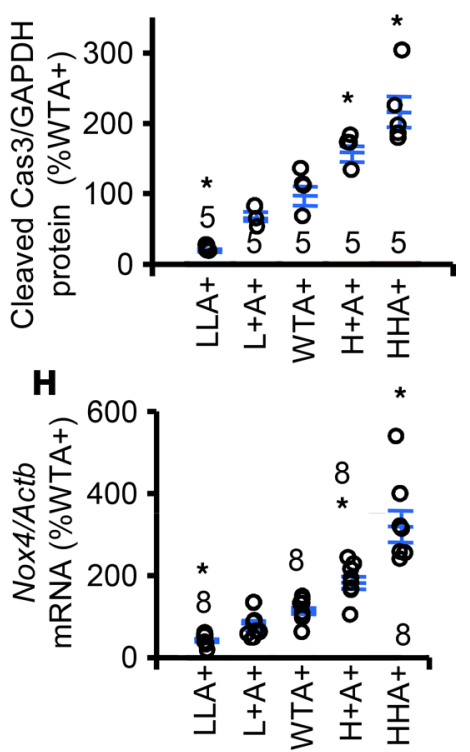

Figure 5. Oxidative stress in the cardiac tissue of Akita diabetic mice. The number of animals studied is shown in each figure. Data are expressed as mean \pm SEM. Comparisons were done with 1-way ANOVA including the additional data set. ${ }^{*} P<0.05$ vs. WTA+ mice by Tukey-Kramer Honestly Significant Differences test. NS, not significantly different among the 5 groups. (A) $\mathrm{H}_{2} \mathrm{O}_{2}$ release. (B) Reduced glutathione (GSH) to oxidized glutathione (GSSC) ratio in the cardiac tissue in Akita diabetic mice with 5 graded expression levels of Elmo1 at 16 weeks of age. (C) An immunoblot for 4-hydroxy-2-nonenal (4-HNE). Forty micrograms of protein was applied to each lane. (D) Overlaid fluorescence of 2-hydroxyethidium $\left(2-\mathrm{OH}^{-} \mathrm{E}^{+}\right)$fluorescence (red), neomycin phosphotransferase II (Neo) immunoreactivity (green), and nuclei attaining with DAPI (blue) in the chimeric hearts of Elmo1//+ Myh6-cre/Esr1 Ins2 ${ }^{\text {Akita/+ }}$ mice. Mice were intraperitoneally injected with low-dose tamoxifen (20 mg/kg, 2 days). The Neo-positive $\mathrm{Elmo}^{\mathrm{L} /+}$ cardiomyocyte ( $($ ) has a lower $2-\mathrm{OH}-\mathrm{E}^{+}$fluorescence than the Neo-negative $\mathrm{Elmo1}^{\mathrm{H/+}}$ cardiomyocyte (\#). Scale bar: $10 \mu \mathrm{m}$. (E) Quantitation of $2-\mathrm{OH}_{-} \mathrm{E}^{+}$by HPLC. (F) Relative amount of cleaved caspase-3 (Cas3). (G) Relative amount of total caspase-3. (H-J) mRNA levels of (H) Nox4, (I) Tgfb1, and (J) Edn1. Amount of mRNA in each sample was normalized by mRNA of $A c t b$ ( $\beta$-actin) and expressed relative to the mean values of WTA+ as $100 \%$.
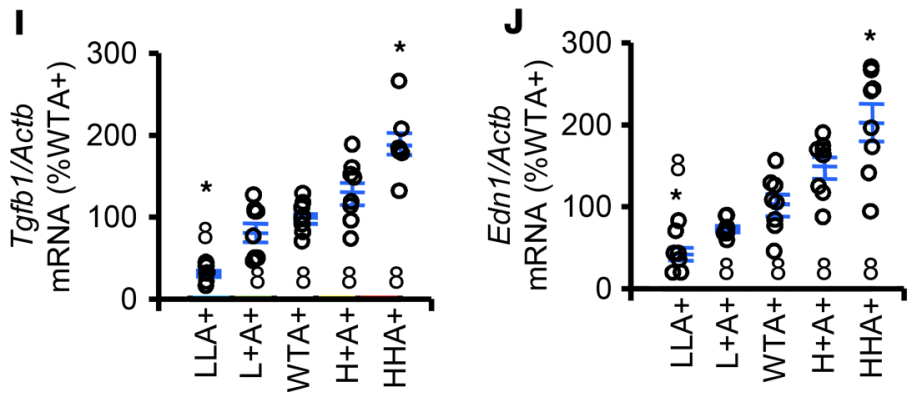

mice than in the LLA+ mice (Figure 4E). Indeed, the mitochondrial number was greater while the mitochondrial size was smaller in the $\mathrm{HHA}+$ and $\mathrm{H}+\mathrm{A}+$ mice than the WTA+ mice (Figure 4, F and $\mathrm{G}$ ). Although the volume density of the mitochondria did not significantly differ (Supplemental Figure 7D), median values of the aspect ratio of mitochondria were smaller in the HHA+ and $\mathrm{H}+\mathrm{A}+$ mice than the WTA+ mice (Supplemental Figure 7E). Total protein levels of mitochondrial fission factor (MFF), which is important for mitochondrial fission, were not different (Supplemental Figure 7F). However, the Ser146-phosphorylated MFF was increased 
A
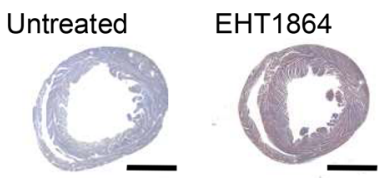

Nox4

VAS3947

B

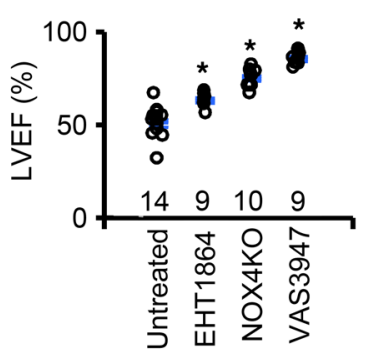

D

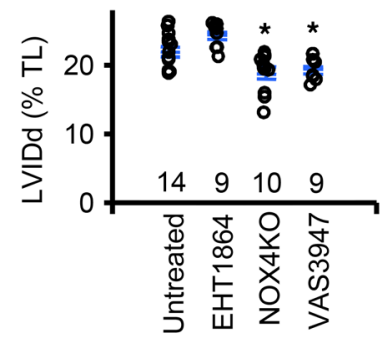

$\mathbf{F}$

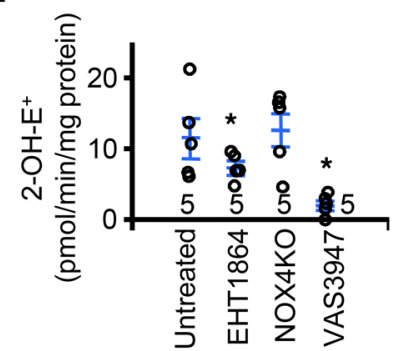

H

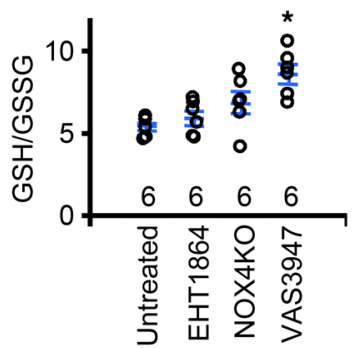

J

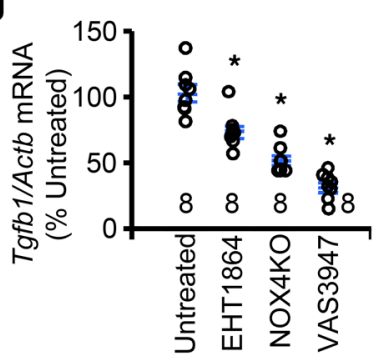

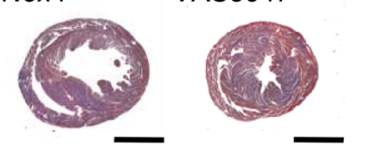

C

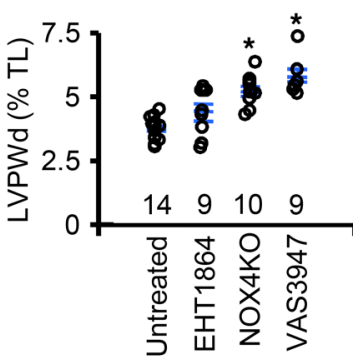

E

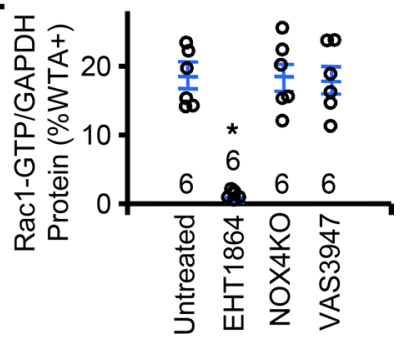

G

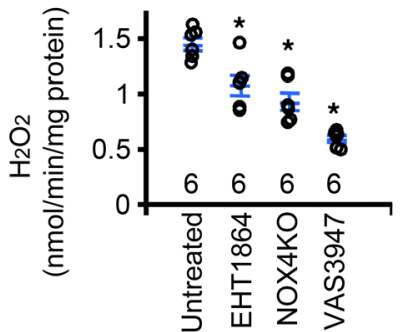

I

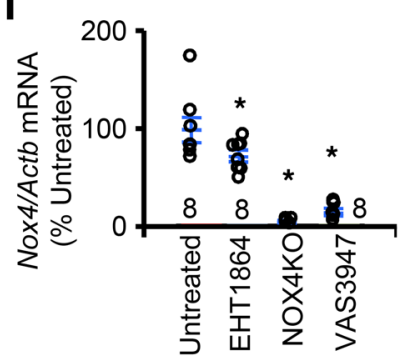

$\mathbf{K}$

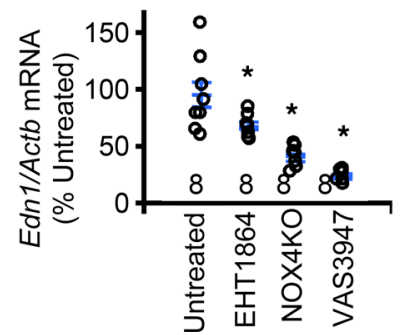

Figure 6. Effects of EHT1864 (a panRac inhibitor), VAS3947 (a pan-NADPH oxidase inhibitor), and disruption of Nox4 (NOX4KO) on the hearts of Akita diabetic mice with high ELM01 expression. All mice were evaluated at 16 weeks of age. Oral treatments with EHT1864 or VAS3947 were given for 4 weeks. Data on the untreated $\mathrm{HHA}+$ mice were taken from the previous experiments. The number of animals analyzed is given in each figure. Data are expressed as mean \pm SEM. Comparisons were done with 1-way ANOVA including the additional data set. ${ }^{*} P<0.05$ vs. untreated HHA+ mice by Tukey-Kramer Honestly Significant Differences test. NS, not significantly different among the 5 groups. (A) AZAN trichrome staining of the hearts. (B) LVEF. (C) Thickness of the LVPWd expressed as percentage of TL. (D) LVIDd expressed as percentage of TL. (E) Rac1 activity. (F) 2-OH-E+ in the cardiac tissue measured by HPLC. (C) $\mathrm{H}_{2} \mathrm{O}_{2}$ release. (H) GSH/GSSG ratio. (I-K) mRNA levels of (I) Nox4, (J) Tgfb1, and (K) Edn1. Amount of mRNA in each sample was normalized by mRNA of $A c t b$ and expressed relative to the mean values of $\mathrm{HHA}+$ mice with vehicle as $100 \%$.

in the Akita mice as the expression of Elmo1 increased (Figure 4H). Similarly, Ser616 phosphorylation, but not total protein, of dynamin-related protein 1, DRP1, was increased as the expression of Elmo1 increased (Figure 4I and Supplemental Figure 7G). Total protein amounts of mitofusin 2 were not altered (Supplemental Figure $7 \mathrm{H})$. Taken together, these results indicate that mitochondrial dysfunction and fragmentation are integral parts of ELMO1-induced exacerbation of cardiac dysfunction in Akita mice. 
ELMO1-mediated cardiac production of ROS. Metabolic dysregulation of diabetes increases cellular production of ROS (14). Furthermore, activated Rac is known to mediate ROS production in a variety of cellular responses in phagocytic as well as nonphagocytic cells, as an essential part of membrane-bound NADPH oxidase complexes (20-23). We previously showed that plasma lipid peroxide levels were increased while erythrocyte glutathione levels were decreased as the expression of Elmo1 increased in Akita diabetic mice (12). In the present study, we also found that the release of hydrogen peroxide and the immunoreactivity of 4-hydroxy-2-nonenal (4-HNE) were increased and the ratio of reduced glutathione (GSH) to oxidized glutathione (GSSG) was decreased in the hearts of Akita mice as the expression of Elmo1 increased (Figure 5, A-C, and Supplemental Figure 8, A and B).

Although the fluorescence after the dihydroethidium injection is not strictly specific to 2-hydroxyethidium converted by superoxide (24), it provided a rough estimation of the intracellular superoxide production in the cardiac cells, which was positively correlated with the amount of ELMO1 in the Akita mice (Figure 5D). For this, we generated Akita mice with the chimeric heart having both $E l m o 1^{L /+}$ and $E l m o 1^{H /+}$ cardiomyocytes by 2-day injections of low-dose tamoxifen into Elmo1 $1^{L /+}$ Myh6-cre/Esr1 Ins $2^{A k i t a /+}$ mice. The treatment converted some Elmo1 $1^{L /+}$ cardiomyocytes to Elmo $1^{H /+}$ cells. The Elmo $1^{L /+}$ cells expressed neomycin phosphotransferase 2 while $E l m o 1^{H /+}$ cells did not, allowing us to distinguish the cells with 2 Elmo1 genotypes of these chimeric hearts. We found that the dihydroethidium-induced fluorescence in the Elmo ${ }^{H /+}$ cells was greater than that in the neighboring Elmo $1^{L /+}$ cells (Figure 5D and Supplemental Figure 8C). This supports that the superoxide production within cells expressing high Elmo1 is greater than that in cells with low Elmo1 expression.

To measure more strictly the amount of superoxide in the hearts of Akita mice with different expression levels of Elmo1, we analyzed 2-hydroxyethidium by separating the oxidation products of dihydroethidium with HPLC (25). The amount of 2-hydroxyethidium was increased as the expression of Elmo1 increased (Figure 5E).

Excess ROS production could damage cellular components and lead to increased cardiomyocyte death, which contributes to the development of cardiomyopathy. Consistent with this possibility, we found that the levels of cleaved caspase-3, a marker for cells that are dying or have died from apoptosis (26), were increased in the hearts of Akita mice as the expression of Elmo1 increased (Figure 5, F-G). Moreover, previous studies reported the involvement of a calcium-activated protease, calpain, in the development of diabetic cardiomyopathy via disrupting ATP synthase and inducing ROS generation $(27,28)$. Calpain activity, measured as ratios of degradation product (BDP145) of $\alpha$ II-spectrin to intact $\alpha$ II-spectrin with and without calcium (29), was enhanced as the Elmol expression increased (Supplemental Figure 8D), suggesting some role for calpain in the ELMO1-linked enhancement of cardiomyopathy.

Although ELMO1 activates Rac, which increases the activity of NADPH oxidases 1 and 2 in the heart, their mRNA levels were not altered (Supplemental Figure 8, E and F). In contrast, mRNA for Rac-independent Nox4 was increased as Elmo1 expression increased (Figure 5H). Although NOX4-specific activity in tissues cannot easily be determined, a direct correlation between the NOX4 mRNA level and enzyme activity has been reported (30). The mRNA and protein levels of genes coding for TGF- $\beta 1$ ( $T g f b 1)$ and endothelin-1 (Edn1), both of which have been shown to induce the expression of Nox4 (31-33), were also increased in parallel with the genetic levels of Elmo1 expression in the Akita mice (Figure 5, I and J, and Supplemental Figure 8, G and $\mathrm{H}$ ). These results indicate that the production of ROS is increased in the hearts of Akita mice overexpressing Elmo1 via the activation of both Rac-dependent and Rac-independent NADPH oxidases.

Pivotal role of NADPH oxidases in the dilated cardiomyopathy in the HHA+ mice. To confirm the involvement of Rac and/or NADPH oxidases in the ELMO1-induced enhancement of diabetic cardiomyopathy, we investigated the effects of 4-week oral administration of EHT1864, a pan-Rac inhibitor, and VAS3947, a pan-NADPH oxidase inhibitor, on the cardiac function in the HHA+ mice starting at the beginning of 12 weeks of age at $40 \mathrm{mg} / \mathrm{L}$ and $4 \mathrm{mg} / \mathrm{L}$, respectively. Neither EHT1864 nor VAS3947 significantly altered plasma glucose levels, systolic blood pressure, renal histology, or plasma cystatin C levels in the HHA+ mice (Supplemental Figure 9, A-F).

EHT1864, partially, and VAS3947, substantially, restored the morphology and systolic and diastolic function in the hearts of the HHA+ mice (Figure 6, A-D, and Supplemental Figure 10, A-D). Chronic oral administration of VAS3947 markedly attenuated both the cardiac systolic and diastolic dysfunctions in the HHA + mice in the PV loop study (Supplemental Figure 10, E-I). It also mitigated the changes in the intercalated discs and restored the immunoreactivity of connexin43, as shown in the TEM of HHA+ hearts (Supplemental Figure 11, A-C). It also mitigated the mitochondrial morphological changes and 
dysfunctions (Supplemental Figure 12, A-G). It also decreased the levels of cleaved caspase-3 in the hearts of HHA+ mice (Supplemental Figure 13, A and B). These data strongly support that the cardiomyopathy of the HHA+ mice is primed by augmented oxidative stress by the enhanced activities of NADPH oxidases as a consequence of the Elmo1 overexpression.

The administered dose of EHT1864 markedly suppressed Rac1 activity (Figure 6E and Supplemental Figure 14A) and significantly decreased cardiac levels of superoxide and $\mathrm{H}_{2} \mathrm{O}_{2}$ to the levels in the diabetic WTA+ mice with normal levels of Elmo1 expression (Figure 6, F and G). EHT1864 did not change cardiac levels of GSH/GSSG significantly (Figure $6 \mathrm{H}$ and Supplemental Figure 14, B and C). In contrast, the effects were more substantial in the VAS3947-treated HHA+ mice, where the levels of these ROS were reduced to levels of the nondiabetic WT mice.

Similarly, EHT1864 partially and VAS3947 substantially decreased cardiac mRNA levels of Nox4, $T g f b 1$, and $E d n 1$ (Figure 6, I-K) and cardiac protein levels of Tgfbl and Edn1 (Supplemental Figure 14, D and E). To further investigate the role of NOX4, the function of which does not require activated Rac1, we generated Nox4-deficient HHA + mice. The Nox4 $4^{-1}$ HHA + mice showed that the disruption of Nox4 significantly alleviated the dilated cardiomyopathy phenotype observed in HHA+ mice (Figure 6, A-K, and Supplemental Figure 10, A-D).

Activated Rac is a well-known regulator of actin polymerization (34), and enhanced actin polymerization has been demonstrated to be associated with impaired cardiac function (35-37). Indeed, actin polymerization was demonstrated to diminish both systolic and diastolic function in the heart $(38,39)$. Consistently, we found that filamentous actin/globular actin ratios in the heart tissues of the Akita mice with graded ELMO1 expression were directly correlated to the ELMO1 expression levels (Supplemental Figure 15). However, the ratios in the hearts of VAS3947-treated HHA+ mice were markedly less than those in untreated $\mathrm{HHA}+$, suggesting that the cytoskeletal change in cardiomyopathy requires NOX-mediated ROS production. Indeed, emerging evidence suggests that actin polymerization is regulated by redox signaling and oxidative stress (40).

Previous studies have also suggested that p21/Rac1-activated kinase 1 phosphorylates the Ser150 residue of cardiac troponin $\mathrm{I}$, which increases the cardiac sensitivity to $\mathrm{Ca}^{2+}$ in a Rac-dependent but ROS-independent fashion (41). The amount of Ser150-phosphorylated cardiac troponin I did not differ significantly among the hearts of Akita mice having 5 levels of ELMO1 (Supplemental Figure 16). Taken together, we conclude that Rac-dependent and Rac-independent NADPH oxidases are the major pathway by which ELMO1 enhances the development of diabetic cardiomyopathy.

\section{Discussion}

In the present study, we conducted a broad characterization of cardiac phenotypes in Akita diabetic mice with 5 different genetically graded levels of Elmo1 expression from $30 \%$ to $200 \%$ of normal. The results demonstrate that ELMO1 is the master regulator of the extent of dysfunction and ultrastructural abnormalities that develop in the diabetic hearts. Twice the normal ELMO1 expression in the HHA+ mice substantially accelerated, while $30 \%$ of normal ELMO1 in the LLA+ mice protected, dilated cardiomyopathy development compared with the WTA+ mice. There were no significant differences in plasma glucose levels or blood pressures among the 5 genotypes or indications of coronary or valvular abnormalities. Furthermore, observed reduction of ejection fraction caused by the cardiomyocyte-specific overexpression of Elmo1 in otherwise ELMO1-hypomorphic Akita mice suggests that the extracardiac (e.g., renal) abnormalities are not required for the development of cardiomyopathy in the ELMO1-hypermorphic, diabetic mice.

The major chain of events leading from ELMO1 to cardiomyopathy is the activation of Rac and of NADPH oxidases. Consistent with previous reports that ELMO1 cooperates with DOCK in activating Rac by exchanging GDP for GTP $(1,2,42)$, activated Rac1 (Rac1-GTP) levels in the hearts ran parallel to ELMO1 levels. The Rac-GTP, among its signaling functions in numerous cellular processes, increases the formation of superoxide anion production by facilitating the assembly of NADPH oxidase complexes (20-23). Indeed, we have reported that genetic increase of ELMO1 in Akita diabetic mice led to progressive increases in the concentrations of circulating oxidative stress markers, including plasma lipid peroxides and erythrocyte GSH levels (12). Furthermore, our current data demonstrate that ELMO1 levels were directly correlated with the tissue levels of superoxide, hydrogen peroxide, GSH, and 4-HNE in the Akita mice as well as the severity of cardiac dysfunction. The core of NADPH oxidase complex is NOX proteins, encoded in the individual Nox genes. NOX1 and NOX2 require the binding of Rac-GTP for their activity and 
are present in the heart. However, we observed that a pan-Rac inhibitor, EHT1864, significantly but only partially decreased the superoxide generation. Attenuation of the cardiac dysfunction and morphological changes in the HHA + mice by EHT1864 was also partial, though significant, suggesting that Rac-dependent NOX does not account for the entire ROS production (Figure 6). In contrast, VAS3947, which blocks ROS generation by inhibiting all isoforms of NADPH oxidases, substantially decreased the superoxide generation and mitigated cardiomyopathy in the HHA+ mice. These findings suggest that a Rac-independent NOX4 (43) also is an important player in ROS generation in the diabetic, ELMO1-hypermorphic mice. We thus found that genetic deficiency of Nox4 significantly, although again not completely, reduced the severity of cardiac dysfunction in the HHA+ mice (Figure 6). The beneficial effect of the Nox4 gene's absence on cardiac function in the ELMO1 HHA+ Akita mice found in the current study is consistent with a previous study showing that the phosphorothioated antisense oligonucleotides for NOX4 prevent cardiac dysfunction in streptozotocin-induced diabetic rats (44). The expression of Nox4 is known to be upregulated in various conditions, including altered shear stress (45), endoplasmic reticulum stress (46), hypoxia (47), and diabetes $(44,48)$, and its mRNA levels directly reflect the NOX4 activities (30). The levels of Nox4 mRNA also paralleled with the Elmo1 expression levels in the hearts of the Akita mice. Previous studies showed that ROS increase the expression of TGF- $\beta 1(49,50)$ and endothelin- $1(51-53)$, both of which are also strong inducers of Nox4 transcription (31-33). Poldip2, a newly discovered Nox4 enhancer protein, could be responsible for the induction of Nox4 in the ELMO1-hypermorphic mice because TGF- $\beta 1$ has been reported to increase the protein levels of poldip2, which enhances the expression and activity of Nox4 (54). In diabetes, where ROS production is enhanced because of metabolic dysregulation, there could exist a vicious circle involving ROS, fibrogenic gene products, and NOX4. Reportedly, a number of kinases, such as p38MAPK and ERK, are activated by ROS (55), in part because of the inhibition of tyrosine phosphatases via oxidized modifications of critical cysteine residues (56), suggesting roles of NOX4-derived ROS in multiple signaling pathways.

The increase in ROS has been suggested as a common element in the multiple mechanisms involved in the development of cardiac disorders in diabetes (57). We observed that systolic and diastolic functions, the frequency of the dissociation of the intercalated disc, reduction in connexin 43 , mitochondrial fragmentation and mitochondrial dysfunction, apoptosis, and actin polymerization were all in direct correlation to ELMO1 levels, supporting that ELMO1 determines the extent of cardiac dysfunction. These features are, however, also correlated directly to the levels of ROS. ROS decrease mitochondrial respiratory function, whereas impaired electron transport chain increases electron leak and leads to excess ROS generation $(58,59)$. This vicious circle could operate in the diabetic heart and develop dilated cardiomyopathy in the ELMO1-hypermorphic Akita mice. More importantly, a pan-NOX inhibitor, VAS3947, spared the HHA+ mice from the development of cardiac dysfunction and morphological features to an extent not different from the LLA+ mice, which are protected from diabetic cardiomyopathy. Except for the direct role of ELMO1 that leads to the ROS production, our literature search found no previous report that suggests a direct involvement of ELMO1 in any of the pathological processes leading to cardiomyopathy. On the other hand, ample evidence has accumulated that excess, prolonged, or out-of-place ROS production can lead to impaired cellular function and pathological conditions (55), even though ROS at normal levels are essential for intracellular cell signaling regulators to maintain normal cell physiology. Cysteine residues are susceptible to oxidation by ROS, and activities of protein tyrosine kinases and protein tyrosine phosphatases are regulated by oxidation of critical cysteine residues (56). This could overactivate or deactivate their downstream signaling cascades. For example, Konishi et al. demonstrated activation of protein kinase C-delta (PKC- $\delta$ ) via tyrosine phosphorylation under oxidative stress (60). PKC- $\delta$ in turn has been shown to phosphorylate Ser616 of DRP1 during oxidative stress and phospho-Ser616-DRP1 promotes mitochondrial fission (61). Similarly, phosphorylation of Ser368 of connexin 43 by PKC- $\delta$ was demonstrated to direct connexin43 away from the gap junction to the protein degradation pathway (62). These previous findings provide potential molecular mechanisms for increased mitochondrial fragmentation and abnormal intercalated discs under conditions of excess ROS production. Together, our observation underscores the importance of ELMO1 as the "rate-limiting" cellular determinant of ROS production, which in turn trigger cellular signaling cascades leading to cardiomyopathy in the heart in diabetes.

Our finding is notable because the cardiopathic effects in diabetes are preventable if Elmo1 expression is genetically decreased to $1 / 3$, even though the diabetic hyperglycemia persists. ELMO1 plays a key pathophysiological role in the development of diabetic complications, largely via overproduction of ROS by both Rac-dependent and Rac-independent NADPH oxidases. The remarkable protective effects of modestly 
decreasing ELMO1 levels and of pan-inhibition of NADPH oxidases in mice suggest that pharmacological suppression of ELMO1 or NADPH oxidases could be promising options for preventing cardiomyopathy in patients with diabetes.

\section{Methods}

Mice. Akita diabetic mice (Ins2 $2^{\text {Akita } /+}$, Jackson Laboratory, 003548) having 5 graded expression levels of Elmo1 were generated by targeted replacement of the 3'-UTR of Elmo1 with an unstable 3'-UTR of a protooncogene, $c F o s\left(E \operatorname{lmo} 1^{L}\right)$, or with a stable $3^{\prime}$-UTR of the bovine growth hormone gene, $b G H$ $\left(E \operatorname{lmo} 1^{H}\right)$, as previously described (12). Conventional PCR was used to determine the genotypes of Elmo1 with the primer sets described in Supplemental Table 2. Akita mice overexpressing Elmo1 specifically in the cardiomyocyte were generated by crossing LLA+ mice with a mouse carrying a tamoxifen-inducible $\alpha$-myosin heavy chain gene (Myho) promoter-driven Cre recombinase transgene (A1cffg(Myhb-cre) $E_{\left.s r 1^{*}\right) / J m k} / \mathrm{J}$, Jackson Laboratory, 005657). Cardiac-specific switch to Elmo1 ${ }^{H / H}$ in otherwise LLA+ mice was achieved using tamoxifen $(20 \mathrm{mg} / \mathrm{kg} / \mathrm{d}$, i.p.; MilliporeSigma) for 5 days at age 12 weeks. Similarly, by injecting a reduced total dose of tamoxifen $(20 \mathrm{mg} / \mathrm{kg} / \mathrm{d}$, i.p., for 2 days $)$ into Elmo1 ${ }^{L /+}$ Myh6-cre Esr1 Ins $2^{\text {Akita/+ }}$ mice at age 12 weeks, we generated male Akita mice with chimeric hearts having both Elmo $1^{L /+}$ and Elmo $1^{H /+}$ cardiomyocytes. One week after the injection of the low-dose tamoxifen, we injected dihydroethidium (50 mg/kg, i.p.; Cayman) 60 minutes before embedding cardiac tissues in optimum cutting temperature compound (Tissue-Plus, Fisher Healthcare) in ethanol and dry ice and storing at $-80^{\circ} \mathrm{C}$ to later compare 2-hydroxyethidium fluorescence between neighboring $E l m o 1^{L /+}$ and $E_{\text {Imo }}{ }^{H /+}$ cardiomyocytes. The mice deficient in NOX4 are from the Jackson Laboratory (no. 022996). An orally available pan-Rac inhibitor, EHT1864 (Adooq; $40 \mathrm{mg} / \mathrm{L}$ in drinking water; ref. 63), and an orally available pan-NOX inhibitor, VAS3947 (MilliporeSigma; $4 \mathrm{mg} / \mathrm{L}$ in drinking water; ref. 64), were administered for 4 weeks beginning at age 12 weeks. Most of the small-molecule inhibitors are not completely specific to the target, and other functions are revealed as they are studied in more detail (65). Nevertheless, VAS3947 is one of the best currently available, which has major effects on inhibition of all NOX enzymes (66). Experimental mice were on a C57BL/6J background, and only male mice were used in this study for all the groups because female Akita mutants do not develop overt diabetes (67). Because of low frequency of obtaining pups with desired genotypes in each litter, multiple crosses of $\mathrm{L}+\mathrm{A}+$ males and $\mathrm{L}+\mathrm{WT}$ females, and multiple crosses of $\mathrm{H}+\mathrm{A}+$ males and $\mathrm{H}+\mathrm{WT}$ females, were used. Consequently, they were not strictly littermates, but phenotypes of WTA + mice from the 2 groups of mating were statistically not different. Experiments were carried out and scoring was done by investigators blinded to the genotype of the animals as much as possible.

Echocardiography, electrocardiography, and PV loop analysis. Heart functions of the conscious mice were analyzed by the Vevo 2100 ultrasonograph system (FUJIFILM VisualSonics) with a 30-MHz transducer. The electrocardiogram was recorded in lead II. All measurements were performed in the University of North Carolina Rodent Advanced Surgical Models Core according to the American Society of Echocardiography guidelines, and the data were analyzed with Vevo 2100 Workstation 1.6.0. software.

For PV loop analysis, mice were anesthetized (ketamine/xylazine 80/10 mg/kg, i.p.), intubated, and placed on a ventilator. A ventral midline skin incision was made, and the thoracic cavity was entered through the sternum. A 1.2-F admittance PV catheter (Sciscence) was introduced into the left ventricle using a 20-gauge needle. After instrumentation was established and PV measurements were obtained, the inferior vena cava was briefly occluded to obtain alterations in venous return for determination of end-systolic and end-diastolic pressure relations. Data analysis was performed with LS-20 LabScribe2 software (iWorx).

Statistics. Data are expressed as mean \pm SEM. To compare groups, we used 1-way or 2-way ANOVA. Post hoc pairwise comparisons were performed by Tukey-Kramer Honestly Significant Differences test (JMP 13; SAS Institute Inc.). A $P$ value less than 0.05 was considered significant.

Detailed methods for measurement of biological parameters, Rac activity/Western blot, calpain activity, quantitative reverse transcription PCR, histology, and immunofluorescence are described in the supplemental methods.

Study approval. All mice were kept under husbandry conditions conforming to the NIH Guide for the Care and Use of Laboratory Animals (National Academies Press, 2011) as approved by the University of North Carolina Institutional Animal Care and Use Committee, Chapel Hill, North Carolina, USA. 


\section{Author contributions}

MK and OS conceived the project and designed experiments. MK, EMB, JRH, RMS, RG, YK, FL, MTS, JCR, and MFA performed experiments. JMT, BAB, JCJ, OS, and NMS helped with project conceptualization and data interpretation. MK, EMB, BAB, and NMS wrote the manuscript with the input of JRH, RG, YK, FL, EYL, MTS, JMT, JCR, MFA, and JCJ.

\section{Acknowledgments}

This work was supported by NIH grants HL49277, HL70523, and KL2TR002490 and by Career Development Award 2006-102 from Juvenile Diabetes Research Foundation. Carolyn B. Suitt (Center for Gastrointestinal Biology and Disease, University of North Carolina at Chapel Hill) prepared heart and kidney sections (P30 DK034987). Kristen K. White, Victoria J. Madden, and Pablo Ariel (Microscopy Services Laboratory, University of North Carolina at Chapel Hill) assisted in light and electron microscopy. We also thank Brian C. Cooley (Animal Surgery Core Laboratory, University of North Carolina at Chapel Hill) for performing the PV loop studies.

Address correspondence to: Masao Kakoki, Department of Pathology and Laboratory Medicine, CB \#7525, 701 Brinkhous-Bullitt Building, University of North Carolina at Chapel Hill, Chapel Hill, North Carolina 27599-7525, USA. Phone: 919.966.6913; Email: mkakoki@med.unc.edu.

1. Brugnera E, et al. Unconventional Rac-GEF activity is mediated through the Dock180-ELMO complex. Nat Cell Biol. 2002;4(8):574-582.

2. Gumienny TL, et al. CED-12/ELMO, a novel member of the CrkII/Dock180/Rac pathway, is required for phagocytosis and cell migration. Cell. 2001;107(1):27-41.

3. Shimazaki A, et al. Genetic variations in the gene encoding ELMO1 are associated with susceptibility to diabetic nephropathy Diabetes. 2005;54(4):1171-1178.

4. Pezzolesi MG, et al. Confirmation of genetic associations at ELMO1 in the GoKinD collection supports its role as a susceptibility gene in diabetic nephropathy. Diabetes. 2009;58(11):2698-2702.

5. Craig DW, Millis MP, DiStefano JK. Genome-wide SNP genotyping study using pooled DNA to identify candidate markers mediating susceptibility to end-stage renal disease attributed to Type 1 diabetes. Diabet Med. 2009;26(11):1090-1098

6. Wu HY, et al. Association of ELMO1 gene polymorphisms with diabetic nephropathy in Chinese population. J Endocrinol Invest. 2013;36(5):298-302.

7. Leak TS, et al. Variants in intron 13 of the ELMO1 gene are associated with diabetic nephropathy in African Americans. Ann Hum Genet. 2009;73(2):152-159.

8. Hanson RL, et al. ELMO1 variants and susceptibility to diabetic nephropathy in American Indians. Mol Genet Metab. 2010;101(4):383-390.

9. Bodhini D, et al. Association of rs11643718 SLC12A3 and rs741301 ELMO1 variants with diabetic nephropathy in South Indian population. Ann Hum Genet. 2016;80(6):336-341.

10. Mehrabzadeh M, et al. Association between ELMO1 gene polymorphisms and diabetic nephropathy in an Iranian population J Diabetes Metab Disord. 2015;15:43.

11. Kakoki M, et al. Altering the expression in mice of genes by modifying their 3' regions. Dev Cell. 2004;6(4):597-606.

12. Hathaway CK, et al. High Elmo1 expression aggravates and low Elmo1 expression prevents diabetic nephropathy. Proc Natl Acad Sci U S A. 2016;113(8):2218-2222.

13. Boudina S, Abel ED. Diabetic cardiomyopathy revisited. Circulation. 2007;115(25):3213-3223.

14. Jia G, Hill MA, Sowers JR. Diabetic cardiomyopathy: an update of mechanisms contributing to this clinical entity. Circ Res. 2018;122(4):624-638

15. Rubler S, Dlugash J, Yuceoglu YZ, Kumral T, Branwood AW, Grishman A. New type of cardiomyopathy associated with diabetic glomerulosclerosis. Am J Cardiol. 1972;30(6):595-602.

16. Veeranki S, Givvimani S, Kundu S, Metreveli N, Pushpakumar S, Tyagi SC. Moderate intensity exercise prevents diabetic cardiomyopathy associated contractile dysfunction through restoration of mitochondrial function and connexin $43 \mathrm{levels} \mathrm{in} \mathrm{db} / \mathrm{db}$ mice. J Mol Cell Cardiol. 2016;92:163-173.

17. Nimlamool W, Andrews RM, Falk MM. Connexin43 phosphorylation by PKC and MAPK signals VEGF-mediated gap junction internalization. Mol Biol Cell. 2015;26(15):2755-2768.

18. Nassal MM, et al. Phosphorylation at Connexin43 serine-368 is necessary for myocardial conduction during metabolic stress J Cardiovasc Electrophysiol. 2016;27(1):110-119.

19. Joo HC, et al. Protective effects of kenpaullone on cardiomyocytes following H. Biochem Biophys Res Commun. 2018;499(2):368-373.

20. Abo A, Pick E, Hall A, Totty N, Teahan CG, Segal AW. Activation of the NADPH oxidase involves the small GTP-binding protein p21rac1. Nature. 1991;353(6345):668-670.

21. Ando S, et al. Post-translational processing of rac p21s is important both for their interaction with the GDP/GTP exchange proteins and for their activation of NADPH oxidase. J Biol Chem. 1992;267(36):25709-25713.

22. Knaus UG, Heyworth PG, Evans T, Curnutte JT, Bokoch GM. Regulation of phagocyte oxygen radical production by the GTP-binding protein Rac 2. Science. 1991;254(5037):1512-1515.

23. Miyano K, Koga H, Minakami R, Sumimoto H. The insert region of the Rac GTPases is dispensable for activation of superoxide-producing NADPH oxidases. Biochem J. 2009;422(2):373-382. 
24. Griendling KK, et al. Measurement of reactive oxygen species, reactive nitrogen species, and redox-dependent signaling in the cardiovascular system: a scientific statement from the American Heart Association. Circ Res. 2016;119(5):e39-e75.

25. Zielonka J, Vasquez-Vivar J, Kalyanaraman B. Detection of 2-hydroxyethidium in cellular systems: a unique marker product of superoxide and hydroethidine. Nat Protoc. 2008;3(1):8-21.

26. Nicholson DW, et al. Identification and inhibition of the ICE/CED-3 protease necessary for mammalian apoptosis. Nature. 1995;376(6535):37-43.

27. Li Y, et al. Targeted inhibition of calpain reduces myocardial hypertrophy and fibrosis in mouse models of type 1 diabetes. Diabetes. 2011;60(11):2985-2994.

28. Ni R, et al. Mitochondrial Calpain-1 disrupts ATP synthase and induces superoxide generation in type 1 diabetic hearts: a novel mechanism contributing to diabetic cardiomyopathy. Diabetes. 2016;65(1):255-268.

29. Romine H, et al. Potential Alzheimer's disease therapeutics among weak cysteine protease inhibitors exhibit mechanistic differences regarding extent of cathepsin B up-regulation and ability to block calpain. Eur Sci J. 2017;13:38-59.

30. Serrander L, et al. NOX4 activity is determined by mRNA levels and reveals a unique pattern of ROS generation. Biochem J. 2007;406(1):105-114.

31. Cucoranu I, et al. $\mathrm{NAD}(\mathrm{P}) \mathrm{H}$ oxidase 4 mediates transforming growth factor-beta1-induced differentiation of cardiac fibroblasts into myofibroblasts. Circ Res. 2005;97(9):900-907.

32. Li Q, Li J, Shao H, Li XX, Yu F, Xu M. Inhibition of CPU0213, a dual endothelin receptor antagonist, on apoptosis via Nox4-dependent ROS in HK-2 cells. Cell Physiol Biochem. 2016;39(1):183-192.

33. Su J, An XR, Li Q, Li XX, Cong XD, Xu M. Improvement of vascular dysfunction by argirein through inhibiting endothelial cell apoptosis associated with ET-1/Nox4 signal pathway in diabetic rats. Sci Rep. 2018;8(1):12620.

34. Nobes CD, Hall A. Rho, rac, and cdc42 GTPases regulate the assembly of multimolecular focal complexes associated with actin stress fibers, lamellipodia, and filopodia. Cell. 1995;81(1):53-62.

35. Heling A, et al. Increased expression of cytoskeletal, linkage, and extracellular proteins in failing human myocardium. Circ Res. 2000;86(8):846-853

36. O'Shea KM, et al. The formin, DIAPH1, is a key modulator of myocardial ischemia/reperfusion injury. EBioMedicine. 2017;26:165-174

37. Schaper J, et al. Impairment of the myocardial ultrastructure and changes of the cytoskeleton in dilated cardiomyopathy. Circulation. 1991;83(2):504-514.

38. Angelone T, et al. Cytoskeleton mediates negative inotropism and lusitropism of chromogranin A-derived peptides (human vasostatin1-78 and rat $\mathrm{CgA}_{1-64}$ ) in the rat heart. Regul Pept. 2010;165(1):78-85.

39. Lin G, et al. Acute inhibition of Rho-kinase improves cardiac contractile function in streptozotocin-diabetic rats. Cardiovasc Res. 2007;75(1):51-58

40. Wilson C, González-Billault C. Regulation of cytoskeletal dynamics by redox signaling and oxidative stress: implications for neuronal development and trafficking. Front Cell Neurosci. 2015;9:381.

41. Buscemi N, Foster DB, Neverova I, Van Eyk JE. p21-activated kinase increases the calcium sensitivity of rat triton-skinned cardiac muscle fiber bundles via a mechanism potentially involving novel phosphorylation of troponin I. Circ Res. 2002;91(6):509-516

42. Sanui T, et al. DOCK2 regulates Rac activation and cytoskeletal reorganization through interaction with ELMO1. Blood. 2003;102(8):2948-2950.

43. Martyn KD, Frederick LM, von Loehneysen K, Dinauer MC, Knaus UG. Functional analysis of Nox4 reveals unique characteristics compared to other NADPH oxidases. Cell Signal. 2006;18(1):69-82.

44. Maalouf RM, et al. Nox4-derived reactive oxygen species mediate cardiomyocyte injury in early type 1 diabetes. Am J Physiol Cell Physiol. 2012;302(3):C597-C604.

45. Hwang J, et al. Pulsatile versus oscillatory shear stress regulates NADPH oxidase subunit expression: implication for native LDL oxidation. Circ Res. 2003;93(12):1225-1232.

46. Santos CX, Tanaka LY, Wosniak J, Laurindo FR. Mechanisms and implications of reactive oxygen species generation during the unfolded protein response: roles of endoplasmic reticulum oxidoreductases, mitochondrial electron transport, and NADPH oxidase. Antioxid Redox Signal. 2009;11(10):2409-2427.

47. Diebold I, Petry A, Hess J, Görlach A. The NADPH oxidase subunit NOX4 is a new target gene of the hypoxia-inducible factor-1. Mol Biol Cell. 2010;21(12):2087-2096.

48. Guo Z, Qin Z, Zhang R, Li J, Yin Y. Effect of rosiglitazone on the expression of cardiac adiponectin receptors and NADPH oxidase in type 2 diabetic rats. Eur J Pharmacol. 2012;685(1-3):116-125.

49. González-Ramos M, et al. Intracellular redox equilibrium is essential for the constitutive expression of AP-1 dependent genes in resting cells: studies on TGF- $\beta 1$ regulation. Int J Biochem Cell Biol. 2012;44(6):963-971.

50. Frippiat C, Chen QM, Zdanov S, Magalhaes JP, Remacle J, Toussaint O. Subcytotoxic $\mathrm{H}_{2} \mathrm{O}_{2}$ stress triggers a release of transforming growth factor-beta 1 , which induces biomarkers of cellular senescence of human diploid fibroblasts. J Biol Chem. 2001;276(4):2531-2537.

51. Hughes AK, Stricklett PK, Padilla E, Kohan DE. Effect of reactive oxygen species on endothelin-1 production by human mesangial cells. Kidney Int. 1996;49(1):181-189.

52. Kaehler J, et al. Endothelin-1 mRNA and protein in vascular wall cells is increased by reactive oxygen species. Clin Sci. 2002;103 Suppl 48:176S-178S.

53. Ruef J, Moser M, Kübler W, Bode C. Induction of endothelin-1 expression by oxidative stress in vascular smooth muscle cells Cardiovasc Pathol. 2001;10(6):311-315.

54. Manickam N, Patel M, Griendling KK, Gorin Y, Barnes JL. RhoA/Rho kinase mediates TGF- $\beta 1$-induced kidney myofibroblast activation through Poldip2/Nox4-derived reactive oxygen species. Am J Physiol Renal Physiol. 2014;307(2):F159-F171.

55. Corcoran A, Cotter TG. Redox regulation of protein kinases. FEBS J. 2013;280(9):1944-1965.

56. Salmeen A, et al. Redox regulation of protein tyrosine phosphatase 1B involves a sulphenyl-amide intermediate. Nature. 2003;423(6941):769-773.

57. Shah MS, Brownlee M. Molecular and cellular mechanisms of cardiovascular disorders in diabetes. Circ Res. 
2016;118(11):1808-1829.

58. Indo HP, et al. Evidence of ROS generation by mitochondria in cells with impaired electron transport chain and mitochondrial DNA damage. Mitochondrion. 2007;7(1-2):106-118.

59. Wu YT, Wu SB, Lee WY, Wei YH. Mitochondrial respiratory dysfunction-elicited oxidative stress and posttranslational protein modification in mitochondrial diseases. Ann N Y Acad Sci. 2010;1201:147-156.

60. Konishi $\mathrm{H}$, et al. Phosphorylation sites of protein kinase $\mathrm{C}$ delta in $\mathrm{H} 2 \mathrm{O} 2$-treated cells and its activation by tyrosine kinase in vitro. Proc Natl Acad Sci U S A. 2001;98(12):6587-6592.

61. Qi X, Disatnik MH, Shen N, Sobel RA, Mochly-Rosen D. Aberrant mitochondrial fission in neurons induced by protein kinase C \{delta $\}$ under oxidative stress conditions in vivo. Mol Biol Cell. 2011;22(2):256-265.

62. Cone AC, et al. Protein kinase C $\delta$-mediated phosphorylation of Connexin 43 gap junction channels causes movement within gap junctions followed by vesicle internalization and protein degradation. J Biol Chem. 2014;289(13):8781-8798

63. Désiré L, et al. RAC1 inhibition targets amyloid precursor protein processing by gamma-secretase and decreases Abeta production in vitro and in vivo. $J$ Biol Chem. 2005;280(45):37516-37525.

64. Hecht N, Terveer N, Schollmayer C, Holzgrabe U, Meinel L. Opening NADPH oxidase inhibitors for in vivo translation. Eur J Pharm Biopharm. 2017;115:206-217.

65. Altenhöfer S, Radermacher KA, Kleikers PW, Wingler K, Schmidt HH. Evolution of NADPH oxidase inhibitors: selectivity and mechanisms for target engagement. Antioxid Redox Signal. 2015;23(5):406-427.

66. Wind S, et al. Comparative pharmacology of chemically distinct NADPH oxidase inhibitors. Br J Pharmacol. 2010;161(4):885-898.

67. Yoshioka M, Kayo T, Ikeda T, Koizumi A. A novel locus, Mody4, distal to D7Mit189 on chromosome 7 determines early-onset NIDDM in nonobese C57BL/6 (Akita) mutant mice. Diabetes. 1997;46(5):887-894. 